\title{
An Enhanced Partial Transmit Sequence Based on Combining Hadamard Matrix and Partitioning Schemes in OFDM Systems
}

\author{
Yasir Amer Al-Jawhar ${ }^{1,3 *}$, Khairun N. Ramli ${ }^{1}$, Montadar Abas Taher ${ }^{3}$, Lukman \\ Audah $^{1}$, Nor Shahida M. Shah ${ }^{2}$, Mustafa S. Ahmed ${ }^{1}$, and Ahmed T. Hammoodi ${ }^{1}$ \\ ${ }^{1}$ Faculty of Electrical and Electronic Engineering, Universiti Tun Hussein Onn Malaysia, Parit Raja, Batu Pahat, 86400, \\ Johor, Malaysia; \\ ${ }^{2}$ Faculty of Engineering Technology, Universiti Tun Hussein Onn Malaysia, Hab Pendidikan Tinggi Pagoh, KM 1, \\ Jalan Panchor, 84600 Panchor, Johor, Malaysia; \\ ${ }^{2}$ Department of Communications Engineering, College of Engineering, University of Diyala, Ba'aqubah, Diyala, \\ 32001, Iraq
}

Received 3 January 2018; accepted 29 April 2018, available online 2 July 2018

\begin{abstract}
The partial transmit sequence (PTS) considered as one of the efficient approaches to restrain the high peak to average-power ratio (PAPR) in orthogonal frequency division multiplexing (OFDM) frameworks. PTS relied on partitioning the input data block and rotate them with a set of the phase vectors. In this study, a novel technique is suggested to improve the PAPR reduction performance in the PTS technique by combining Hadamard matrix and the popular kinds of the partitioning schemes interleaving scheme (IL-PTS), adjacent scheme (AdPTS), and pseudo-random scheme (PR-PTS). The new approach employed Hadamard matrix to change some of the subcarrier phases of the partitioning scheme in the frequency-domain. The simulation results demonstrated that the new method improved the PAPR diminishment performance better than that of the PR-PTS and Ad-PTS. However, the proposed method achieved the same PAPR performance compared with the IL-PTS scheme.
\end{abstract}

Keywords: PTS, PAPR, OFDM, PR-PRS, Ad-PTS, IL-PTS

\section{Introduction}

OFDM is an effective technology for the high-speed data rate in wireless communication system because of the prominent features of the OFDM system such as the simplicity of implementation [1], durability against multipath fading channels [2], and efficiency of bandwidth utilization [3]. Accordingly, many communication frameworks depended on the OFDM systems as a transmission data technology such as digital video broadcasting (DVB) [4], wireless local area network (WLAN) IEEE.802.11 [5], and worldwide interoperability for microwave access IEEE.802.16 [6]. In addition, the fourth generation $(4 \mathrm{G})$ frameworks utilized OFDM as a data transmission system in the standard Long-Term Evaluation (LTE) [7]. On the other hand, the high PAPR is the major shortcoming of the OFDM system, which leads to the spectrum deterioration in transmission side [8]. If the system is run with high PAPR, some components of the system, for example, high power amplifiers will be worked out the scope of the linear region, and this leads to out-of-band radiation and in-band distortion. Hence, the system suffers from bit error rate degradation and spectrum inefficiency.

Several approaches have been suggested to mitigate the high PAPR value, for example, clipping and filtering [9], Partial transmit sequence (PTS) [10], selective- mapping (SLM) [11], and interleaving technique [12]. PTS is considered as one of the influential approaches to improve the PAPR mitigation performance compared with the other probabilistic techniques [13]. The PTS method depends on two parts of its procedure, partitioning the input data sequence and changing the phases of the partitioned data sequence. In literature, many scenarios have been proposed to enhance the PAPR alleviation performance in terms of developing the partitioning schemes such as Jawhar [14][15] proposed several methods to improve the PAPR lessening performance better than the ordinary partitioning approaches. Also, Ibraheem [16] and Hong [17] proposed new methods to diminish the PAPR value better than the conventional PTS methods by combining two types of the ordinary segmentation schemes.

The target of this paper is PAPR lessening performance improvement better than the conventional PTS algorithm. The proposed method integrates Hadamard matrix with the partitioning schemes (H-PTS) in the frequency-domain to change the high consistency phases of the input data sequence. The results indicated that the PAPR diminishing performance of the new method outperforms to the PR-PTS and Ad-PTS schemes. However, the proposed approach achieves the same performance of the IL-PTS method. 


\section{OFDM Framework}

In OFDM, the input data sequence $X$ one of the mapping techniques such as quadrature amplitude modulation (QAM) modulates in order to generate the baseband signal in the frequency domain. Next, the data signal is converted from the serial into parallel pattern. Therefore, the baseband signal can be described as

$$
X_{k}=\left[X_{0}, X_{1}, \ldots \ldots, X_{N-1}\right]^{\mathrm{T}}
$$

where $N$ represents the number of subcarriers. After that, the inverse fast Fourier transform (IFFT) is applied to the baseband signal to produce the discrete time domain signal $x(n)$, which can be defined as [18]

$$
x(n)=\frac{1}{\sqrt{N}} \sum_{k}^{N-1} X_{k} e^{j 2 \pi k \frac{n}{N}} \quad, 0 \leq n \leq N-1
$$

where $j=\sqrt{-1}$. In the time domain, the OFDM signal is generated by superposition the baseband signals so some peaks power of the subcarriers may be added together and then will become much larger than the mean power when the subcarriers have the same phases. This degradation named PAPR, and can be written as [19]

$$
\operatorname{PAPR}=\frac{\max |x(n)|^{2}}{E\left\{|x(n)|^{2}\right\}}
$$

where $E\{$.$\} symbolizes the mean power. The$ complementary-cumulative-distribution-function (CCDF) is utilized to evaluate the probability of the PAPR that surpasses a certain threshold value and can be written as [20]

$$
\mathrm{P}_{\mathrm{r}}\left(\operatorname{PAPR}\left(x(n)>P A P R_{0}\right)=1-\left(1-e^{-P A P R_{0}}\right)^{N}\right.
$$

where $P A P R_{0}$ represents the threshold value. Moreover, The PAPR calculations will be more accurate, if the oversampling operation is applied. This operation is done by inserting $(L-1) N$ zeros between the OFDM subcarriers [21], where $L$ is the oversampling factor. Accordingly, the CCDF of the OFDM signal can be established as

$$
\mathrm{P}_{\mathrm{r}}\left(\operatorname{PAPR}\left(x(n)>\mathrm{PAPR}_{0}\right)=1-\left(1-e^{-\mathrm{PAPR}_{0}}\right)^{N L}\right.
$$

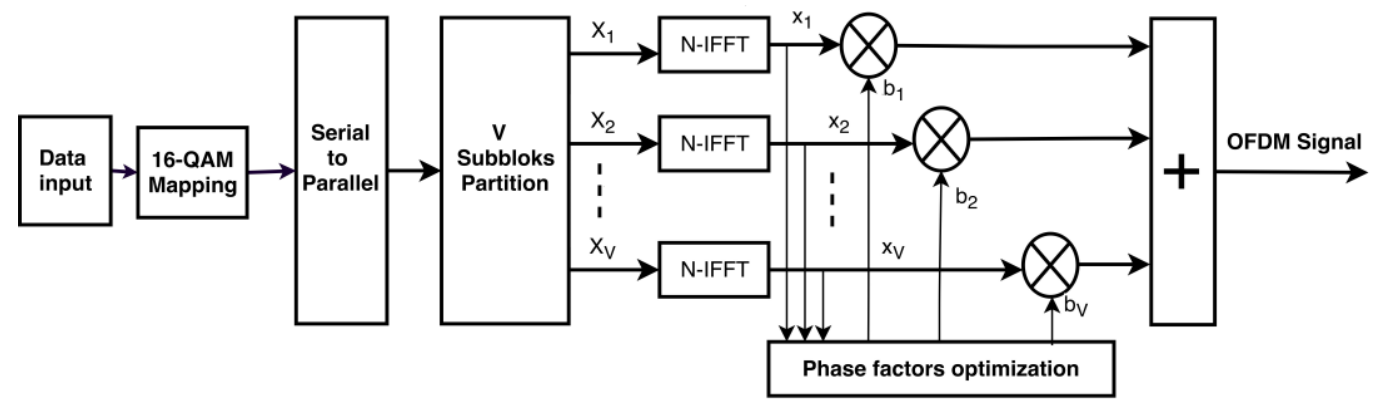

Fig. 1: PTS block diagram [22]

\section{Partial transmit sequence (PTS)}

As can be observed in Figure 1, PTS procedure starts when partitioning the baseband sequence into $V$ subblocks before applying $N$-IFFT. Consequently, the partitioned baseband signal can be demonstrated as

$$
X=\sum_{v=1}^{V} X_{v}
$$

After that, the subblocks is rotated by set of the phase rotation factors $\left(b_{v}\right)$, so the OFDM signal in the time domain is defined as

$$
x(n)=\operatorname{IFFT}\left\{\sum_{v=1}^{V} b_{v} X_{v}\right\}
$$

The linear property of the IFFT is applied to the $\left(b_{v}\right)$, so the OFDM signal in the time-domain can be written as

$$
x(n)=\sum_{v=1}^{V} b_{v} \operatorname{IFFT}\left\{X_{v}\right\}
$$

and,

$$
x(n)=\sum_{v=1}^{V} b_{v} x_{v}
$$

The elements of the phase rotation factors can be obtained as

$$
b_{v}=e^{j 2 \pi v / W}, \quad\{v=0,1, \ldots \ldots, W-1\}
$$

where $W$ expresses the number of the allowed phase factors, which is usually limited in order to avoid more of the complex multiplication operations, so $b_{v} \in\{ \pm 1\}$ or $\{ \pm 1, \pm \mathrm{j}\}$ [22]. In the time-domain, every subblock is weighted by phase rotation vectors $b_{v}$, and combines with other subblocks to produce a set of candidate signals. The PAPR is calculated for each candidate, and the candidate signal that has the minimum PAPR is elected for transmission. Accordingly, the system must check $\left(W^{V-1}\right)$ groups of the phase factors to find the optimum phase rotation factor; with the consideration that the first 
element of the phase factors $b_{1}$ usually determines to 1 , without any influence on the PAPR performance. Moreover, the transmitter side should send $\left(\log _{2} W^{V-1}\right)$ bits to the receiver in order to ensure recovering the original data, so this operation limits the bandwidth of the system [23].

\section{PTS partitioning schemes}

In PTS, there are three popular forms of the partitioning schemes including interleaving scheme, adjacent scheme and, pseudo-random scheme, as demonstrated in Fig. 2. Each one of the partitioning schemes has a PAPR reduction performance different of another depending on the correlation between the subcarriers [24]. In IL-PTS, $N / V$ of the subcarriers is assigned with a constraint interval of $V$ for each subcarrier within subblock. However, the Ad-PTS scheme assigns $N / V$ consecutive subcarriers inside each subblock, successively. On the same hand, the PR-PTS scheme allocates $N / V$ of the subcarriers randomly inside each subblock. All the partitioning schemes must fulfil the following condition; the sub-blocks must be equaled in size and non-overlapping with each other.
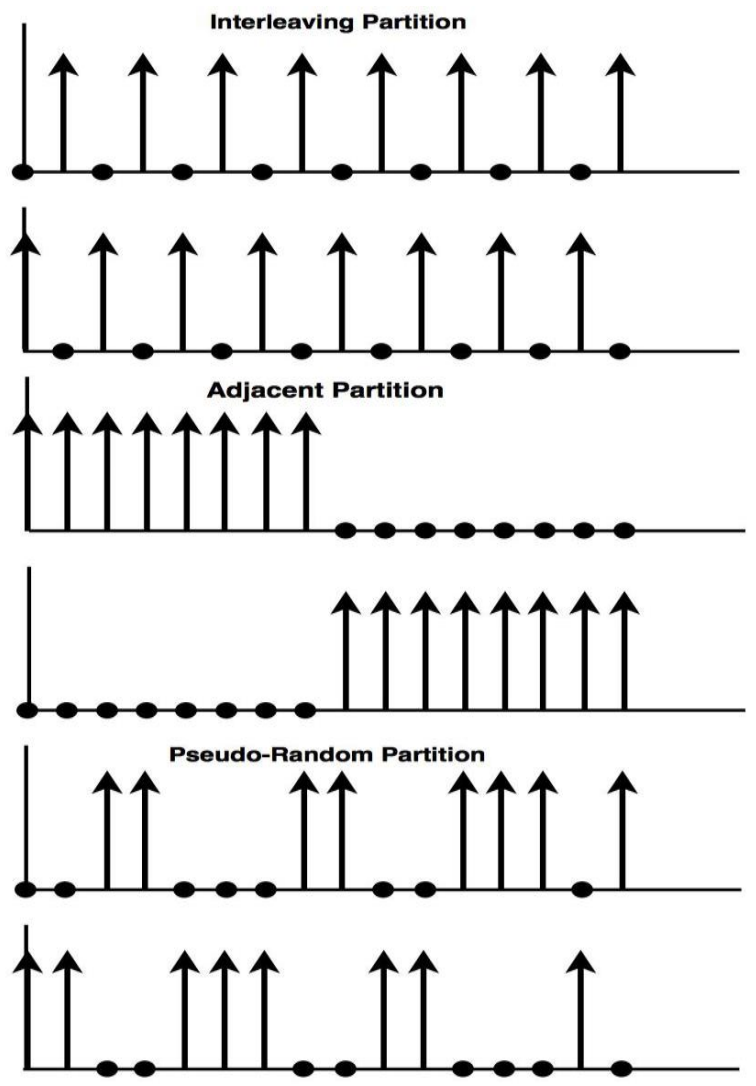

Fig. 2 Conventional partitioning schemes [22]

In addition, the PAPR detraction performance of the PR-PTS is the best among the three partitioning schemes, whereas the Ad-PTS scheme is the second best. However, the IL-PTS is considered the worst partitioning scheme concerning of the PAPR reduction [25]. In contrast, the PR-PTS and Ad-PTS have computational complexity higher than that of the IL-PTS scheme.

\section{Hadamard matrix}

Hadamard matrix (H matrix) is a square matrix, and its elements either +1 or -1 . Moreover, the rows of the $\mathrm{H}$ matrix are orthogonal to each other [26]. The low order of the $\mathrm{H}$ matrix can be written as

$$
\mathrm{H}_{1}=[1], \quad \mathrm{H}_{2}=\left[\begin{array}{ll}
+1 & +1 \\
+1 & -1
\end{array}\right]
$$

Likewise, the $\mathrm{H}$ matrix can be established by

$$
H_{2 M}=\left[\begin{array}{ll}
H_{M} & H_{M} \\
H_{M} & -H_{M}
\end{array}\right]
$$

where $\mathrm{M}$ is a decimal number.

\section{Hadamard-PTS method}

The contribution of this paper is the achievement of PAPR lessening performance better than that of the conventional PTS algorithm, so the Hadamard-PTS method (H-PTS) is proposed to enhance the PAPR lessening performance. The H-PTS approach integrates $\mathrm{H}$ matrix with PTS partitioning scheme in the frequencydomain, in which the $\mathrm{H}$ matrix is produced depending on the subblocks number $V$, as follow

$$
\mathrm{H}_{V}=\left[\begin{array}{ll}
\mathrm{H}_{V / 2} & \mathrm{H}_{V / 2} \\
\mathrm{H}_{V / 2} & -\mathrm{H}_{V / 2}
\end{array}\right]
$$

After that, the $\mathrm{H}_{V}$ matrix is duplicated relying on the number of the subcarriers $\mathrm{N}$, so the $\mathrm{H}_{V}$ matrix is repeated $(N / V)$ times to produce the $\mathrm{HD}_{r}$ matrix, which can be defined as

$$
\mathrm{HD}_{r}=\left[\mathrm{H}_{V, 1}, \mathrm{H}_{V, 2}, \ldots, \mathrm{H}_{V, N / V}\right]
$$

where $r=\{1,2, \ldots, N / V\}$, and represents the duplication times, this operation ensures the length of the rows of the Hadamard matrix equal to that of the partitioning scheme matrix. Afterwards, the $\mathrm{HD}_{r}$ matrix is multiplied by the petitioning scheme matrix to produce a new partitioning scheme matrix (H-PTS), as follow

$$
\mathrm{H}-\mathrm{PTS}=\mathrm{HD}_{r}^{\mathrm{T}} * X_{V, N}^{\mathrm{T}}
$$

where $X_{V, N}^{\mathrm{T}}$ represents the conventional partitioning scheme matrix. Finally, the rest of the PTS procedure is 
applied to the H-PTS scheme, and the OFDM sequence that achieves the minimal PAPR value is chosen for transmission.

In summary, the PAPR alleviation performance of the H-PTS algorithm is enhanced, because the $\mathrm{H}$ matrix can reduce the autocorrelation between the subcarriers within the subblocks [27]. Equations (16-19) illustrate the $\mathrm{H}$ matrix integration with Ad-PTS (H-Ad-PTS) method, as example, where the number of subblocks $V$ is chosen to four, and the number of subcarriers $N$ is chosen to eight. The $\mathrm{H}_{V}$ matrix is duplicated to produce $\mathrm{HD}_{r}$ matrix, which is multiplied by the Ad-PTS matrix. As can be seen in H-Ad-PTS matrix, the signs of the Ad-PTS matrix are changed depending on the corresponding elements of the $\mathrm{HD}_{r}$ matrix. Accordingly, the proposed method works to alter the phases of the subcarriers within the subblocks. Hence, the PAPR decreasing performance of the proposed method will be improved.

The $\mathrm{H}_{V}$ matrix can be obtained by

$$
\mathrm{H}_{V}=\left[\begin{array}{llll}
+1 & +1 & +1 & +1 \\
+1 & -1 & +1 & +1 \\
+1 & +1 & -1 & -1 \\
+1 & -1 & -1 & +1
\end{array}\right]
$$

The $\mathrm{HD}_{r}$ matrix can be produced by

$$
\mathrm{HD}_{r}=\left[\mathrm{H}_{V, 1}, \mathrm{H}_{V, 2}\right]=\left[\begin{array}{rrrrrrrr}
+1 & +1 & +1 & +1 & +1 & +1 & +1 & +1 \\
+1 & -1 & +1 & -1 & +1 & -1 & +1 & -1 \\
+1 & +1 & -1 & -1 & +1 & +1 & -1 & -1 \\
+1 & -1 & -1 & +1 & +1 & -1 & -1 & +1
\end{array}\right]
$$

The Ad-PTS matrix can be written as

$$
X_{V, N}=\left[\begin{array}{cccccccc}
X_{1,1} & X_{1,2} & 0 & 0 & 0 & 0 & 0 & 0 \\
0 & 0 & X_{2,3} & X_{2,4} & 0 & 0 & 0 & 0 \\
0 & 0 & 0 & 0 & X_{3,5} & X_{3,6} & 0 & 0 \\
0 & 0 & 0 & 0 & 0 & 0 & X_{4,7} & X_{4.8}
\end{array}\right]
$$

Therefore, the H-Ad-PTS matrix $=\mathrm{HD}_{r}^{\mathrm{T}} * X_{V, N}^{\mathrm{T}}$, and can be expressed as

$$
\text { H-Ad-PTS }=\left[\begin{array}{cccccccc}
X_{1,1} & X_{1,2} & 0 & 0 & 0 & 0 & 0 & 0 \\
0 & 0 & X_{2,3} & -X_{2,4} & 0 & 0 & 0 & 0 \\
0 & 0 & 0 & 0 & X_{3,5} & X_{3,6} & 0 & 0 \\
0 & 0 & 0 & 0 & 0 & 0 & -X_{4,7} & X_{4.8}
\end{array}\right]
$$

\section{Results and Discussion}

In this part, the proposed method is simulated and compared with the conventional partitioning schemes. The $\mathrm{H}$ matrix is integrated with PR-PTS, Ad-PTS, and IL-PTS to produce H-PR-PTS, H-Ad-PTS, and H-IL-PTS schemes, respectively. The parameters of this simulation are: the number of subblocks $V=4$, the number of subcarriers $N=128$ and 256 , the number of the different phase rotation factors $W=2$, and the oversampling factor $L$ is fixed to 4 . Moreover, 16-QAM is employed to generate 1000 random symbols.

Fig. 3 and Fig. 4 demonstrates the comparison between the H-PR-PTS method and PR-PTS method when $N=128$ and 256 . The PAPR value of the original OFDM signal (without PTS) was $10.79 \mathrm{~dB}$, and the PAPR value of the PR-PTS method achieved $8.208 \mathrm{~dB}$, while the PAPR value of the H-PR-PTS method fulfilled $7.945 \mathrm{~dB}$, as shown in Fig. 3. The simulation result as demonstrated in Fig. 4 indicated that the H-PR-PTS method achieved the superiority to the PR-PTS method concerning the PAPR reduction performance by $0.263 \mathrm{~dB}$ when $N=256$. Therefore, the H-PR-PTS scheme can achieve greater PAPR detraction performance than that of the PR-PTS scheme in both scenarios.

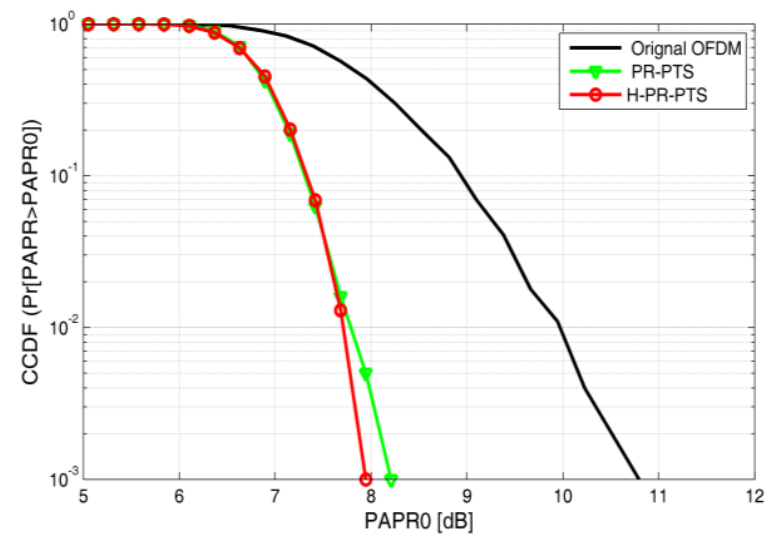

Fig. 3 Comparison between the H-PR-PTS and PR-PTS when $N=128$

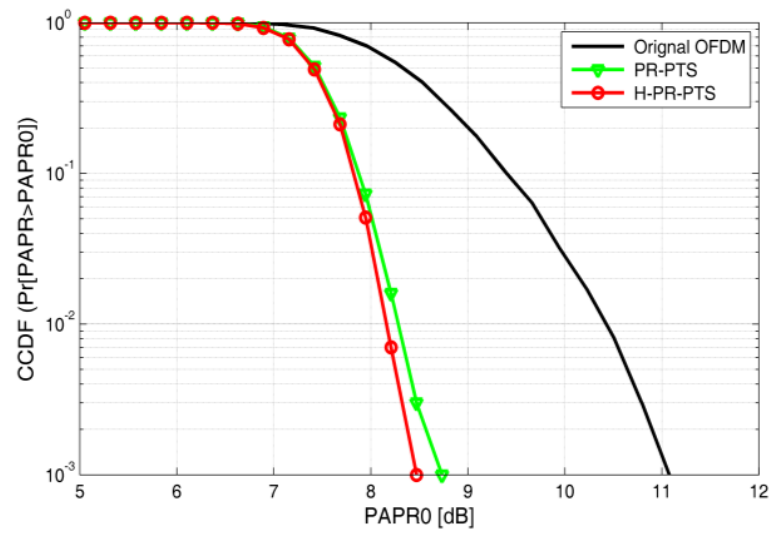

Fig. 4 Comparison between the H-PR-PTS and PR-PTS when $N=256$

As can be observed in Fig. 5 and Fig. 6, the H-AdPTS algorithm was compared with the Ad-PTS algorithm when $N=128$ and 256 . It is clearly seen that the H-AdPTS can be exceeded the Ad-PTS by $0.36 \mathrm{~dB}$ when $N=128$. Also, H-Ad-PTS accomplished the superiority to the Ad-PTS by $0.4 \mathrm{~dB}$ when $N=256$. Consequently, the PAPR detraction performance of the H-Ad-PTS 
outperforms to the Ad-PTS in any number of the subcarriers.

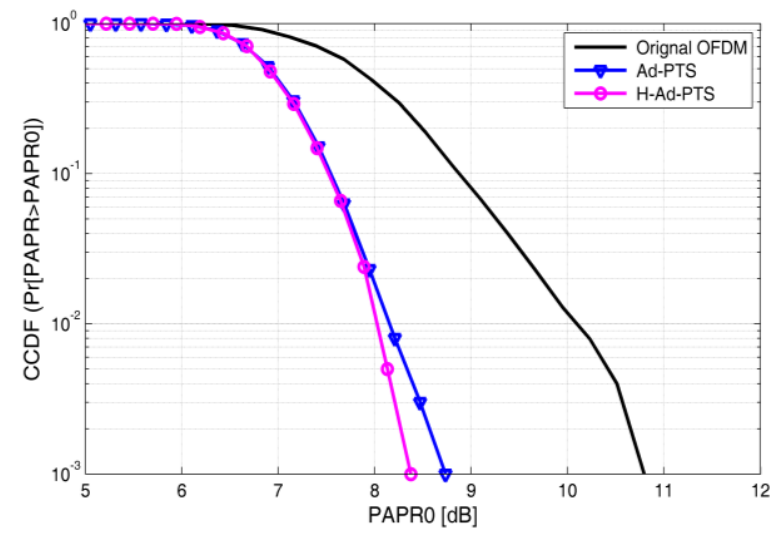

Fig. 5 Comparison between the H-Ad-PTS and Ad-PTS when $N=128$

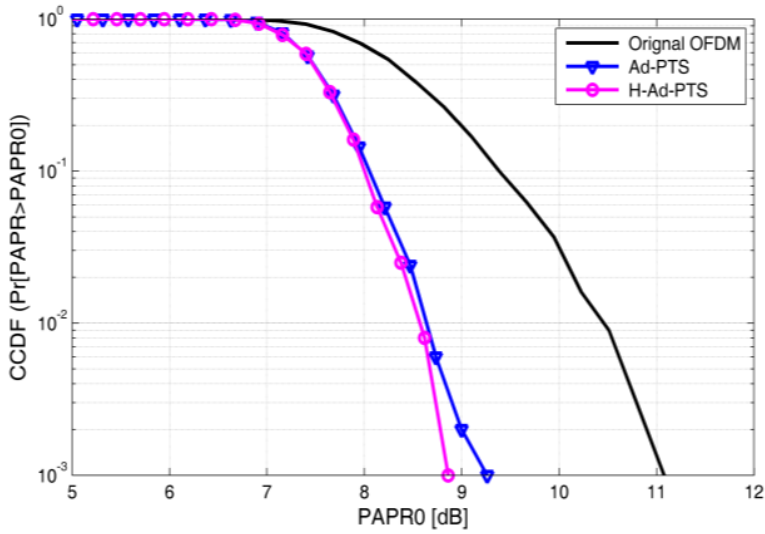

Fig. 6 Comparison between the H-Ad-PTS and Ad-PTS when $N=256$

On the other hand, the comparison of the H-IL-PTS scheme and IL-PTS scheme is conducted when the number of the subcarriers is 128 and 256, as demonstrated in Fig. 7 and Fig. 8. In both scenarios, the H-IL-PTS and IL-PTS have the same value of the PAPR, $9.06 \mathrm{~dB}$ when $N=128$, and $9.566 \mathrm{~dB}$ when $N=256$. Consequently, the H-IL-PTS cannot enhance the PAPR decreasing performance higher with IL-PTS scheme.

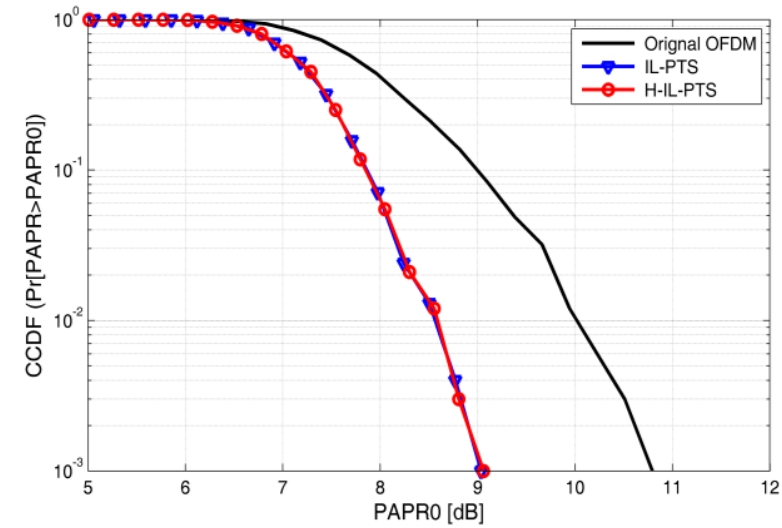

Fig. 7 Comparison between the H-IL-PTS and IL-PTS when $N=128$

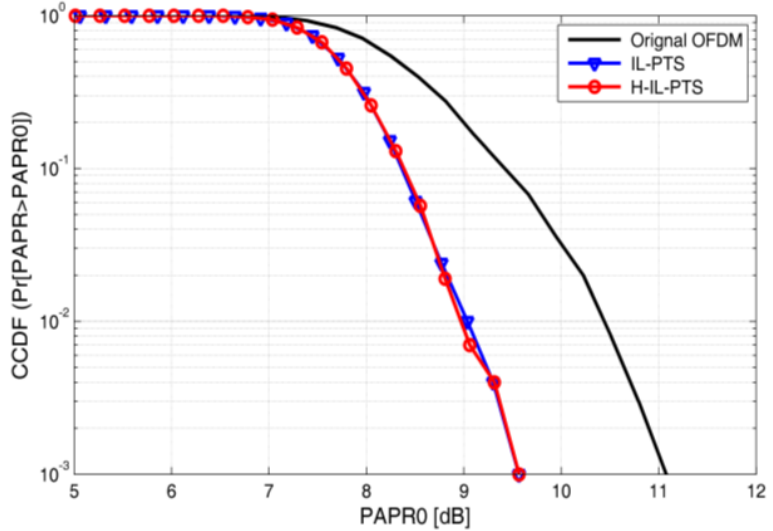

Fig. 8 Comparison between the H-IL-PTS and IL-PTS when $N=256$

According to the simulation results above, the $\mathrm{H}$ matrix can affect to the PR-PTS and Ad-PTS schemes, because the structure matrix of these schemes is nonperiodic, so the $\mathrm{H}$ matrix works to change some phases of the subcarriers in these schemes. Hence, the H-PR-PTS and H-Ad-PTS schemes outperform to the PR-PTS and Ad-PTS schemes in PAPR reduction. In contrast, the HIL-PTS approach achieved the same PAPR diminishing performance of IL-PTS scheme. This can be attributed to that the IL-PTS matrix is a periodic matrix so that the $\mathrm{H}$ matrix cannot change the phases of subcarriers in IL-PTS matrix. Hence, the PAPR performance of the H-IL-PTS scheme is similar to that of the IL-PTS matrix.

In general, the proposed methods can slightly improve the PAPR reduction performance compared with PR-PTS and Ad-PTS segmentation schemes, this can be attributed of that the Hadamard matrix elements contains either +1 or -1 , thereby in each row of the Hadamard matrix, the elements which have negative signs (-1) will change the phases of corresponding subcarriers of the PR-PTS matrix or Ad-PTS matrix by $180^{\circ}$. Depending on the OFDM operation, the high PAPR value of the output OFDM signal is due to superposition $N$ subcarriers at the output of IFFT in the transmitter; with the condition the phases of these subcarriers are the same value. Therefore, the Hadamard matrix works to improve the PAPR performance by changing the phases of the subcarriers.

The simulation results in Fig 3, Fig 4, Fig 5, and Fig 6 show the PAPR reduction values about $0.3 \mathrm{~dB}$ in $\mathrm{H}-\mathrm{PR}$ PTS and about $0.4 \mathrm{~dB}$ in H-Ad-PTS. The one can be observed that the PAPR reduction performance is slightly improved, but this improvement considers good if we compared the performance with the PR-PTS which is well-known as the best PAPR reduction method among the conventional segmentation schemes. Also, the Hadamard matrix changed some of the subcarrier phases depending on the (-1) locations in the matrix. Hence, the phases of these subcarriers influence positively to the PAPR lessening performance. Moreover, the Hadamard can be saved in the receiver memory, and it can be easily removed from the OFDM signal when recovering the original data in the receiver side. In other words, the proposed methods do not need to send extra side 
information to receiver side to inform it of the changes by Hadamard matrix. Therefore, the proposed methods improved the PAPR reduction performance better than that of the ordinary methods without additional complexity.

\section{Summary}

A novel method has been proposed in this paper named H-PTS; this method accomplished PAPR diminishing performance better than that of the PR-PTS and Ad-PTS schemes in PTS technique. The new method works to combine Hadamard matrix with partitioning scheme of the PTS to produce a new scheme which can change the phases of the subcarriers inside the subblocks in the frequency-domain. The new approach is applied to the three well-known types of the partitioning schemes. The simulation results indicated that the H-PR-PTS and H-Ad-PTS methods outperform to the PR-PTS and AdPTS schemes in terms of PAPR reduction because the influence of the Hadamard matrix on the partitioning schemes led to changing the phases of the subcarriers of the partitioning schemes and then the PAPR detaction performance is improved. Nevertheless, the H-IL-PTS method achieved the same PAPR decreasing performance of the IL-PTS, because the Hadamard matrix cannot affect to the periodic scheme as the IL-PTS scheme. Therefore, the proposed method considers simple execution algorithm to improve the PAPR mitigation performance in PTS technique.

\section{Acknowledgements}

This paper was partly sponsored by Center for Graduate Studies. The researchers are grateful to Universiti Tun Hussein Onn Malaysia (UTHM) for sponsoring this work and supporting further from the Center of Graduate Student (CGS).

\section{References}

[1] Rahmatallah, Y., and Mohan, S. Peak-to-Average Power Ratio Reduction in OFDM Systems: A Survey and Taxonomy. IEEE communications surveys \& tutorials, Volume 4(15), (2013), pp. 1567-1592.

[2] Chen, Z., and Kang, S. A Three-dimensional OFDM System with PAPR Reduction Method for Wireless Sensor Networks. International Journal of Distributed Sensor Networks, (2014), pp 1-6. Available online at: https:// doi.org/10.1155/2014/312308

[3] P. Praveenkumar, R. Amirtharajan, K. Thenmozhi, and Rayappan J. OFDM with Low PAPR: A Novel Role of Partial Transmit Sequence. Research Journal of Information Technology, 1(5), (2013), pp. $35-44$.

[4] Jayashri R., Sujatha, S. and Dananjayan, P. DCT Based Partial Transmit Sequence Technique for PAPR Reduction in OFDM Transmission. ARPN
Journal of Engineering and Applied Sciences, Volume 10(5), (2015), pp. 2182-2186.

[5] Jawhar, Y., Abdulhasan, R. and Ramli, K. Influencing Parameters in Peak to Average Power Ratio Performance on Orthogonal FrequencyDivision Multiplexing System. ARPN Journal of Engineering and Applied Sciences, Volume 6(11), (2016), pp. 4322-4332.

[6] Alhasson, B. and Matin, M. PAPR Distribution Analysis of OFDM Signals with Partial Transmit Sequence. In 4th International Conference on Computer and Information Technology (ICCIT), (2011), pp: 652-656.

[7] Barideh, M. Improving the Performance of Generalized Frequency Division Multiplexing (GFDM) Modulaion Scheme by Using Walish Hadamard Transform (WHT) Pre-Coded. Journal of Engineering and Applied Sciences, Volume 11(12), (2016), pp. 2755-2763.

[8] Müller, S., and Huber, J. A Novel Peak Power Reduction Scheme for OFDM. In 8th IEEE International Symposium on Personal, Indoor and Mobile Radio Communications (PIMRC'97), (19997), pp: 1090-1094.

[9] Taher, M., Mandeep, J., Ismail, M., Samad, S., and Islam, M. Reducing the Power Envelope Fluctuation of OFDM Systems Using Side Information Supported Amplitude Clipping Approach. International Journal of Circuit Theory and Applications, Volume 4(42), (2014), pp. 425-435.

[10] Müller, S., and Huber, J. OFDM with Reduced PeakTo-Average Power Ratio by Optimum Combination of Partial Transmit Sequences. IEEE Electronics letters, Volume 5(33), (1997), pp. 368-369.

[11] Taher, M., Mandeep, J., Ismail, M., Samad, S., and Islam, M. Post-IFFT-Modified Selected Mapping to Reduce the PAPR of an OFDM System. Circuits, Systems, and Signal Processing, Volume 34(2), (2015), pp. 535-555.

[12] Han, S., and Lee, H. An Overview of Peak-ToAverage Power Ratio Reduction Techniques for Multicarrier Transmission. IEEE Wireless Communications, Volume 2(12), (2005), pp. 56-65.

[13] Müller, S., and Huber, J. A Comparison of Peak Power Reduction Schemes for OFDM. In IEEE conference on Global Telecommunications (GLOBECOM'97), (1997), pp. 1-5

[14] Jawhar, Y., Ahmad, M. Abdulhasan, R., Hamzah, S., and Ramli, K. A New Hybrid Sub-Block Partition Scheme of PTS Technique for Reduction PAPR Performance in OFDM System. ARPN Journal of Engineering and Applied Sciences, Volume 7(11), (2016), pp. 3904-3910.

[15] Jawhar, Y., Shah, N., Taher, M. Ahmed, M., and Ramli, K. An Enhanced Partial Transmit Sequence Segmentation Schemes to Reduce the PAPR in OFDM Systems. International Journal of Advanced Computer Science and Applications (IJACSA). Volume 7(12), (2016), pp. 66-75. 
[16] Ibraheem, Z., Rahman, M., Yaakob, S., Razalli, M., Salman, F., and Ahmed, K. PTS Method with Combined Partitioning Schemes for Improved PAPR Reduction in OFDM System. Indonesian Journal of Electrical Engineering and Computer Science, Volume 11(12), (2014), 7845-7853.

[17] Hong, C., Qin, Q., and Chao, T. A PTS Optimization Algorithm for PAPR Reduction of OFDM System. In Proceeding International Conference on Mechatronic Sciences, Electric Engineering and Computer (MEC), (2013), pp. 3775-3778.

[18] Kim, K. On the Shift Value Set of Cyclic Shifted Sequences for PAPR Reduction in OFDM Systems. IEEE Transactions on Broadcasting, Volume 2(62): (2016), pp. 496-500.

[19] Mhatre, K. and Khot, U. Efficient Selective Mapping PAPR Reduction Technique. International Conference on Advanced Computing Technologies and Applications (ICACTA-2015) Procardia Computer Science, (2015), pp. 620-627.

[20] Chisab, F. Comparative Study in Performance for Subcarrier Mapping in Uplink 4G-LTE under Different Channel Cases. IJACSA International Journal of Advanced Computer Science and Applications, Volume 5, (2014), pp. 46-54.

[21] Tellambura, C. Computation of the ContinuousTime PAR of an OFDM Signal with BPSK Subcarriers. IEEE Communications Letters, Volume 5(5), (2001), 185-187.

[22] Jawhar, Y., Shah, N., Taher, M. Ahmed, M., and Ramli, K. and Abdulhasan, R. A Low PAPR Performance with New Segmentation Schemes of Partial Transmit Sequence for OFDM Systems. International Journal of Advanced and Applied Sciences, Volume 4(4), (2017), pp. 14-21.

[23] Wu, X., Wang, J., Mao, Z., and Zhang, J. Conjugate Interleaved Partitioning PTS Scheme for PAPR Reduction of OFDM Signals. Circuits, Systems and Signal Processing, Volume 3(29), (2010), pp. 499514.

[24] Xia, L., Yue, X., Youxi, T., and Shaoqian, L. A Novel Method to Design Phase Factor for PTS Based on Pseudo-Random Sub-Block Partition in OFDM System. In 66th IEEE Vehicular Technology Conference (VTC-2007), (2007), pp: 1269-1273.

[25] Kang, S., Kim, J., and Joo, E. A Novel Subblock Partition Scheme for Partial Transmit Sequence OFDM. IEEE Transactions on Broadcasting, Volume 3(45): (2001), pp. 333-338.

[26] Park, M., Jun, H., Cho, j., Cho, N., Hong, N., and Kang, C. PAPR Reduction in OFDM Transmission Using Hadamard Transform. In IEEE International Conference on Communications (ICC 2000), (2000), pp: 430-433.

[27] Miao, L., and Sun, Z. PTS Algorithm for PAPR Suppression of WOFDM System. International Conference on Mechatronic Sciences, Electric Engineering and Computer (MEC), (2013), pp. 1144-1147. 